\title{
Network specific change in white matter integrity in mesial temporal lobe epilepsy
}

\section{AUTHOR(S):}

Imamura, Hisaji; Matsumoto, Riki; Takaya, Shigetoshi; Nakagawa, Tomokazu; Shimotake, Akihiro; Kikuchi, Takayuki; Sawamoto, Nobukatsu; ... Fukuyama, Hidenao; Takahashi, Ryosuke; Ikeda, Akio

\section{CITATION:}

Imamura, Hisaji ...[et al]. Network specific change in white matter integrity in mesial temporal lobe epilepsy. Epilepsy Research 2016, 120: 65-72

\section{ISSUE DATE:}

2016-02-01

URL:

http://hdl.handle.net/2433/210294

\section{RIGHT:}

C2015. This preprint version is made available under the CC-BY-NC-ND 4.0 license http://creativecommons.org/licenses/by-nc-nd/4.0/; This is not the published version. Please cite only the published version.; この論文は出版社版でありません。引用の際には 出版社版をご確認ご利用ください。 
Network specific change in white matter integrity in mesial temporal lobe

epilepsy.

Hisaji Imamura ${ }^{\text {1 }}$, Riki Matsumoto ${ }^{\text {, Shigetoshi Takayac, f, g, Tomokazu Nakagawa }}$, Akihiro Shimotake $^{\mathrm{a}}$, Takayuki Kikuchi ${ }^{\mathrm{d}}$, Nobukatsu Sawamoto ${ }^{\mathrm{a}}$, Takeharu Kunieda ${ }^{\mathrm{d}}$, Nobuhiro Mikunid, e, Susumu Miyamotod, Hidenao Fukuyamac, Ryosuke Takahashia ${ }^{a}$ Akio Ikeda ${ }^{\mathrm{b}}$

a. Department of Neurology, Kyoto University Graduate School of Medicine, 54 Shogoin Kawahara-cho, Sakyo-ku, Kyoto, 606-8507, Japan

b. Department of Epilepsy, Movement Disorders and Physiology, Kyoto University Graduate School of Medicine, 54 Shogoin Kawahara-cho, Sakyo-ku, Kyoto, 606-8507, Japan

c. Human Brain Research Center, Kyoto University, 54 Shogoin Kawahara-cho, Sakyo-ku, Kyoto, 606-8507, Japan

d. Department of Neurosurgery, Kyoto University Graduate School of Medicine, 54 Shogoin Kawahara-cho, Sakyo-ku, Kyoto, 606-8507, Japan

e. Department of Neurosurgery, Sapporo Medical University, S1 W17, Chuo-ku, 
Sapporo, 060-8556, Japan

f. Radioisotope Research Center, Kyoto University, Kyoto, 606-8315, Japan

g. A. Martinos Center for Biomedical Imaging, Massachusetts General Hospital, Harvard Medical School, MA 02129, USA

Present address

1. Hisaji Imamura, Department of Neurology, Fukui Red Cross Hospital, 4 Tsukimi, Fukui, 918-8501, Japan

E-mail

Hisaji Imamura, imamurah@kuhp.kyoto-u.ac.jp

Riki Matsumoto, matsumot@kuhp.kyoto-u.ac.jp

Shigetoshi Takaya, shig.t@kuhp.kyoto-u.ac.jp

Tomokazu Nakagawa, tnakaga@kuhp.kyoto-u.ac.jp

Akihiro Shimotake, smtk@kuhp.kyoto-u.ac.jp

Takayuki Kikuchi, tkik@kuhp.kyoto-u.ac.jp

Nobukatsu Sawamoto, sawa@kuhp.kyoto-u.ac.jp

Takeharu Kunieda, kuny@kuhp.kyoto-u.ac.jp

Nobuhiro Mikuni, mikunin@sapmed.ac.jp 
3

Susumu Miyamoto, miy@kuhp.kyoto-u.ac.jp

Hidenao Fukuyama, fukuyama@kuhp.kyoto-u.ac.jp

Ryosuke Takahashi, ryosuket@kuhp.kyoto-u.ac.jp

Akio Ikeda, akio@kuhp.kyoto-u.ac.jp

Corresponding to: Riki Matsumoto \& Akio Ikeda, Kyoto University Graduate School

of Medicine, 54 Shogoin Kawahara-cho, Sakyo-ku, Kyoto, 606-8507, Japan

Tel \& fax: $\quad$ +81-75-751-3772 \& +81-75-751-9416

E-mail: matsumot@kuhp.kyoto-u.ac.jp (RM), akio@kuhp.kyoto-u.ac.jp (AI)

Title: 83 characters

Text: 20 pages, 4192 words

5 figures, 1 table, 1 supplementary figure 


\section{Summary}

Objectives: To identify the specific change of white matter integrity that occurs in the brain network related to epileptic activity in patients with mesial temporal lobe epilepsy

(MTLE).

Methods: We recruited 18 patients with MTLE and 18 healthy subjects. In MTLE patients, the remote functional-deficit zone was delineated using fluorodeoxyglucose positron emission tomography as an extratemporal region showing glucose hypometabolism. Using diffusion magnetic resonance imaging tractography, we defined a seizure propagation tract (PT) as a white matter pathway that connects the focus with a remote functional deficit zone. We also used the corticospinal tract (CST) and inferior longitudinal fasciculus (ILF) as control tracts in the hemisphere ipsilateral to the focus. Fractional anisotropy (FA), mean diffusivity (MD), and volume of the tracts were compared among PT, CST, and ILF.

Results: Tractographic analysis identified the uncinate fasciculus, arcuate fasciculus, and fornix as PTs. A decrease in FA was found in MTLE patients compared with healthy subjects in all tracts, but PTs showed a more significant decrease in FA than did the two control tracts. Although the change in MD was also found in MTLE patients compared with healthy controls, a tract-specific change was not observed. Although white-matter damage was observed in all candidate tracts examined, the integrity of white matter was 
most significantly decreased in PTs in MTLE.

Conclusion: The change in white matter integrity occurs specifically in the pathways that connect the focus and remote functional deficit zones in patients with MTLE, i.e., the pathways that are assume to be associated with seizure propagation. 


\section{Introduction}

1.1 In patients with mesial temporal lobe epilepsy (MTLE), glucose hypometabolism often extends beyond the temporal lobe. Among them, glucose hypometabolism in the prefrontal cortex is associated with cognitive changes (Jokeit et al., 1997; Takaya et al., 2006), suggesting that functional deficit zones exist in the remote cortical regions (Rosenow and Luders, 2001).

1.2 Glucose hypometabolism in the remote cortical regions from the epileptic focus reflects the preferential networks involved by ictal discharges and is also associated with seizure frequency (Chassoux et al., 2004; Takaya et al., 2006). In addition, after the epileptogenic lesion in the mesial temporal lobe is removed, the remote areas that supposedly receive projections from the affected area show improved glucose metabolism (Dupont et al., 2001; Spanaki et al., 2000; Takaya et al., 2009). These lines of evidence suggest that the functional deficit zones in remote cortical regions are most likely the result of the frequent propagation of epileptic activity from the epileptic focus through a white matter pathway.

1.3 Previous studies using voxel-wise whole brain analysis of white matter integrity such as tract-based spatial statistics (TBSS) have shown that the change in white matter integrity occurs throughout the brain in patients with MTLE (Focke et al., 2008; 
Schoene-Bake et al., 2009; Yogarajah et al., 2010). One possible reason is that inherent pre-existing abnormalities due to genetic factors or developmental abnormalities might exist in the whole brain in patients with MTLE (Velisek and Moshe, Epilepsia 2003; Love, Lancet Neurol 2005). However, whether there is any pathway that is specifically impaired compared with other white matter pathways in patients with MTLE remains unclear. Given that the epileptic activity arising from the focus is assumed to generate the functional deficit zone in remote cortical regions through white matter pathways as mentioned above, we would hypothesize that epileptic activity specifically impairs these pathways.

1.4 In this study, we first delineated the white matter pathways that connect the focus and the remote functional deficit zones in MTLE patients by combining FDG-PET and diffusion MRI tractography. We traced these specific white matter pathways into the cortices that showed glucose hypometabolism in FDG-PET by adopting probabilistic diffusion tractography that can trace beyond region of high uncertainty into the grey matter or across the crossing fibers (Behrens et al., 2007). Then we evaluated the change in white matter integrity of these pathways using tractography-based regions of interest (ROI) method. The advantage of this method is that it allows the cross-subject comparison of white matter integrity along the given tracts without requiring cross-subject registration (Smith et al., 2013). We expected to see that the pathway that connects the focus and the 
remote functional deficit zones is affected much more than other white matter pathways.

Our findings may provide insight into the wide range of functional network impairment in patients with localization-related epilepsy.

\section{Methods}

\subsection{Subjects}

2.1.1 We recruited 18 right-handed patients with medically intractable MTLE (10 with left MTLE and 8 with right; mean age, 30.7 years; ranging, 19-45 years; 8 men, 10 women). All patients underwent presurgical evaluation between 2007 and 2010 at the Kyoto University Hospital. These patients were diagnosed as MTLE on the basis of seizure semiology, electroencephalography (EEG), video EEG monitoring, and neuroimaging data. We detected hippocampal atrophy or sclerosis in all 18 MTLE patients by means of 3-tesla MRI. Patients with additional MRI anomalies outside the mesial temporal lobe were excluded from this study. Eight patients underwent surgery. Ten patients are on a waiting list for surgery. The details of the patients' demographic data are shown in table 1 . We also analyzed 18 age-matched right-handed healthy control subjects (mean age, 31.3 years; ranging, 18-47 years; 10 men, 8 women). 


\subsection{Standard Protocol Approvals, Registrations, and Patient Consents.}

\subsubsection{This study was approved by the Ethics Committee of Kyoto University Graduate}

School of Medicine (approval\# E-430), and written informed consent was obtained from all patients and healthy volunteers.

\subsection{FDG-PET data analysis}

2.3.1 The FDG-PET scans were performed using a PET scanner (Advance, General Electric Medical System, Milwaukee, WI, USA). [18F]-FDG at 370 MBq (10mCi) was injected intravenously into subjects who had been fasting for at least four hours. Then, 40 minutes after the administration of the radiotracer, 35 slices of brain-emission images were acquired over a 20 -min period. The subjects were studied in an awake, resting state, with their eyes closed and ears unplugged in a dimly lit environment. Although EEG was not recorded during FDG-PET study, it was unlikely an ictal study because no abnormal behaviors were observed, and because patients did not report any subjective manifestations of seizure during the examination. Emission images were reconstructed into a $128 \mathrm{x} 128$ matrix image with a pixel size of $1.95 \times 1.95 \mathrm{~mm}^{2}$ and slice thickness of $4.25 \mathrm{~mm}$. All reconstructed images were corrected for attenuation using ${ }^{68} \mathrm{Ge}-{ }^{68} \mathrm{Ga}$ transmission scans 
performed before the actual scan. The FDG-PET images from the patients with right

MTLE were flipped so that the epileptic focus was on the left side in all images. Images from the same number of healthy volunteers were also flipped. Voxelwise analysis of the FDG-PET images was performed using Statistical Parametric Mapping 8 (SPM8). The FDG-PET images were coregistered with the anatomical three-dimensional (3D) MRI images of each person by means of the mutual information algorithm implemented in SPM8. The flipped FDG-PET images of the patients with right MTLE and the same number of healthy volunteers were coregistered with the flipped MRI images. The coregistered FDG-PET images were then spatially normalized to fit an in-house FDG-PET template using affine and nonlinear warping. The spatially normalized images were smoothed using an isotropic Gaussian kernel with 16-mm full width at half maximum (FWHM) to increase the signal-to-noise ratio and to account for normal interindividual macroanatomical variation. To eliminate the effects of global neuronal activity, each voxel count was normalized to the total count of the whole brain using proportional scaling.

2.3.2 A two-sample $t$ test was used for the voxelwise group comparison of the FDG-PET images between patients and healthy volunteers. Adjustment for gender as a confounder was performed by using it as a nuisance variable. We analyzed the brain regions showing a decrease in glucose metabolism at the height threshold $(p=0.001$; 
uncorrected for multiple comparison) and an extent threshold of 300 voxels. Thus, a hypometabolic region, i.e., a functional deficit zone was identified in the MTLE group in the Montreal Neurological Institute (MNI) standard space (figure 1A). The hypometabolic region was observed within the following two regions: 1 ) the temporal lobe ipsilateral to the focus, i.e., the anterior lateral and basal temporal areas, which included both the hippocampus and parahippocampus that were thought to be the epileptic focus and 2) the ipsilateral extratemporal area, i.e., the ventrolateral prefrontal area.

\subsection{MRI data acquisition}

2.4.1 Magnetic resonance images were acquired on a 3-T Siemens Trio scanner.

Diffusion weighted image (DWI), dual-gradient field map, and three-dimensional (3D) anatomical image were acquired using a 3.0 Tesla MRI scanner (Trio, Siemens, Erlangen, Germany) with an eight-channel phased-array head coil. DWI was acquired using single-shot spin-echo echo-planar sequence. The scanning parameters as follows: echo time $(\mathrm{TE})=96 \mathrm{~ms}$; repetition time $(\mathrm{TR})=10,500 \mathrm{~ms}$; flip angle $(\mathrm{FA})=90$; field of view $($ FOV $)=192 \times 192 \mathrm{~mm} 2$; slices $=70$; voxel size $=2 \times 2 \times 2 \mathrm{~mm}$. The diffusion weighting was isotropically distributed along 81 directions using a b value of $1,500 \mathrm{~s} / \mathrm{mm} 2$. Nine volumes of no diffusion weighting ( $b=0 \mathrm{~s} / \mathrm{mm} 2$ ) were also acquired at points throughout 
the acquisition. Dual-gradient field map in an axial orientation was obtained for the b0

unwarping of DWI using the following parameters: TR $=511 \mathrm{~ms}$; TE1/TE2 $=$ 5.19/7.65

$\mathrm{ms} ; \mathrm{FA}=60^{\circ} ; \mathrm{FOV}=192 \times 192 \mathrm{~mm}$; slices $=46$; voxel size $=3 \times 3 \times 3 \mathrm{~mm}$. The scanning

parameters of T1-weighted three-dimensional anatomical images [Magnetization Prepared

Rapid Gradient Echo, (MPRAGE)] were as follows: TE = 4.38 ms; TR = 2,000 ms;

176 $\times 292$ matrix; FOV = $176 \mathrm{~mm} \times 292 \mathrm{~mm} 2$; slice of $1.0 \mathrm{~mm}$ thickness. The details of

acquisition of diffusion-weighted images (DWI), a dual-gradient field map, 3D anatomical

images, and postprocessing of the DWI data (Diffusion tensor imaging and probabilistic

diffusion tractography) have been reported elsewhere (Oguri et al., 2013; Yamao et al., 2014).

\subsection{Tractography-based assessment of white matter integrity along the tracts}

2.5.1 We herein defined a putative propagation tract (PT) of epileptic activity as a white matter tract that connects the epileptic focus and a remote functional deficit zone. As control tracts, we selected the inferior longitudinal fasciculus (ILF) and the corticospinal tract (CST). The ILF was chosen because both the PT and the ILF usually originate from the anterior and basal temporal areas (Catani et al., 2003), whereas the target cortical region is different (the frontal functional deficit zone versus the occipital lobe). The CST 
was selected as a tract that was away from the epileptic focus in the temporal lobe. Both control tracts were reported to show good reproducibility during identification of tracts using diffusion tractography (Wakana et al., 2007).

2.5.2 In order to identify the PT, the seed region of interest (ROI) was placed in the hypometabolic temporal area that comprised the epileptic focus. This is partly because of a technical limitation: it is difficult to perform diffusion tractography directly on the hippocampus because diffusion-weighted signals are often distorted there because of air susceptibility artifacts. Moreover, atrophy was present in the MTLE group. From the standpoint of the "epileptogenic zone”(Rosenow and Luders, 2001), it is also difficult to confine the focus to the hippocampus alone. The epileptogenic zone in MTLE is not always restricted to the hippocampus but occasionally is present in the temporal pole and the basal part (Coste et al., 2002; Moran et al., 2001). Furthermore, according to other FDG-PET studies, the hypometabolic temporal area surrounding the hippocampus is related to generation of ictal discharge and spread pathways (Chassoux et al., 2004). According to these pieces of evidence, in the present tractography study, we decided to place the seed ROI in the hypometabolic temporal region. The target ROI of PT was placed in the frontal hypometabolic region that represented the extratemporal functional deficit zone. To divide the hypometabolic region into the temporal and frontal subregions, we 
manually set a 3-mm-thick boundary between the two subregions at the border of the two lobes ( $3 \mathrm{~mm}$ thick; a white mask in figure 1B). These ROIs were set up in the MNI standard space. For the left-MTLE patients and the same number of randomly assigned healthy volunteers, these ROIs were transferred to the T1 anatomical space in each subject using the inverted transformation matrices of spatial normalization. For the right-MTLE patients and the rest of healthy volunteers, we flipped the ROIs in the MNI space so that they were located in the right hemisphere, and then we transferred them to the T1 anatomical space in each individual brain.

2.5.3 In each brain, we segmented the gray matter of the seed and target ROIs using FMRIB's Automated Segmentation Tool of the FSL software (fsl.fmrib.ox.ac.uk). These segmented, cortical portions of the seed and target ROIs served as the seed and target masks, respectively. To exclude erroneous pathways such as the tracts going into the contralateral hemisphere, exclusion masks were placed on the midline sagittal plane and the boundary between the two hypometabolic subregions (white masks in figure 1B). In the two control tracts (CST and ILF), the seed and target masks were set up according to the methods reported by Wakana ${ }^{12}$ with modifications (details in the appendix). The seed mask for inferior longitudinal fasciculus (ILF) was set at the same seed mask as used for tracing PT, namely, the segmented temporal hypometabolic region. The target ROI was set 
in the ipsilateral occipital lobe defined by the MNI atlas implemented in FSLView software.

This target ROI was transferred onto T1 anatomical space in each subject using the inverted transformation matrices of spatial normalization. On a coronal directional color-coded map, an additional waypoint mask was placed in the coronal plane passing through the caudal end of the splenium of the corpus callosum. The tracts that were situated lateral or inferior to the lateral ventricle and running in the anterior-posterior direction were selected. The exclusion mask was set on a midline sagittal plane.

2.5.4 Regarding Cortico-Spinal-Tract (CST), the seed mask was manually set in the cerebral peduncle ipsilateral to the focus by using the axial directional color-coded map in each subject. The target mask was set in the ipsilateral precentral gyrus of Harvard Oxford Atlas (www.cma.mgh.harvard.edu). The exclusion mask was set at a midline sagittal plane. 2.5.5 These target and exclusion masks were constructed in the MNI standard space, and then transformed onto each subject's brain (MPRAGE) using the inversion transformation matrices of spatial normalization. Those made in the directional color-coded map in each subject's brain were also linearly coregistered onto each subject's MPRAGE. Probtracx function of FDT was performed using these masks in MPRAGE space.

2.5.6 Probabilistic diffusion tractography was performed for the PT and control tracts 
(ILF and CST) with the aforementioned seed, target, and exclusion masks using FMRIB's

Diffusion Toolbox (FDT) of the FSL software. Probability density functions were

calculated by drawing 5,000 samples from each voxel in the seed mask. The connectivity

value was the number of samples that passed through a voxel; this number was

automatically calculated by the probtrackx function of FDT. In contrast to deterministic

streamlining tractography, we needed a certain threshold value to extract significant

tractography findings. Because no gold standard exists for the selection of the threshold,

we traced these three tracts using thresholds $0.1 \%, 1 \%, 5 \%, 10 \%$, and $20 \%$ of the

maximum connectivity value calculated separately for each tract. We then calculated mean

fractional anisotropy (FA), mean diffusivity (MD), and volume of the tracts at each

threshold. Because the seed masks of PT and ILF were identical in the temporal lobe, we

masked out the tracts within the temporal hypometabolic region (both the gray- and white

matter) for measurement of FA and MD of the PT and ILF.

2.5.7 FA, MD, and volume of the tracts were subjected to repeated-measures analysis of variance (ANOVA), with the tract (PT versus ILF versus CST) as a within-subject factor, and the group (MTLE patients versus healthy volunteers) as a between-subject factor. If there was a tract-by-group interaction among the three tracts, we performed post hoc analysis of all three pairs (PT versus CST, PT versus ILF, and CST versus ILF). Statistical 
significance was set at $\mathrm{p}<0.05$. We used the SPSS 15.0J software for statistical

calculations.

\subsection{Voxel-wise whole brain assessment of white matter integrity}

2.6.1 We also assessed white matter integrity using TBSS to see the change that occurs throughout the whole brain in MTLE patients that has shown in previous studies (Focke et al., 2008; Schoene-Bake et al., 2009; Yogarajah et al., 2010). In brief, TBSS projects all subjects' FA data onto a mean FA tract skeleton, before applying voxelwise cross-subject statistics. Both control minus patient and patient minus control contrasts were tested, with 5,000 permutations. Multiple comparisons were corrected using a threshold-free cluster enhancement (TFCE) (Smith et al. 2009). Voxelwise statistical inference was made on these output images, and significance level was set at $\mathrm{p}<0.01$, corrected for family-wise error rate.

\section{Results}




\subsection{The putative propagation tract}

3.1.1 It was traced from the temporal to the frontal functional deficit zone at several thresholds $(0.1 \%, 1 \%, 5 \%, 10 \%$, and $20 \%)$. Because of the "probabilistic" nature of the tractography, as the threshold increased, the traced tracts changed from a widespread distribution to more restricted or focused one (figure 2). At the threshold $0.1 \%$, too many tracts were uncovered to extract the core PT. At the $1 \%$ threshold, three pathways were detected. The first pathway ran through the uncinate fasciculus (UF) toward the prefrontal cortex. The second pathway ran through the long segment of the arcuate fasciculus (AF). The third pathway first ran through the fornix to the thalamus and ended in the prefrontal cortex (figure 2). When the threshold was $\geq 5 \%$, UF and AF became the predominant pathways among these tracts. At the $20 \%$ threshold, only UF remained significant among the three pathways. The seed mask encompassed the gray matter of the whole temporal hypometabolic region for both PT and ILF, but the actual seed areas of the UF and ILF overlapped and were confined to the anterior part of the parahippocampal and fusiform gyri (figure 5).

\subsection{Comparison of white matter integrity in the propagation tract and control tracts}

\subsubsection{The FA values of PT (FAPT), ILF (FAILF), and CST (FACST) were compared}


between the MTLE and healthy control groups. All three tracts showed decreased FA in

MTLE patients. Repeated-measures ANOVA demonstrated a significant interaction

between the tract (PT, IFL, or CST) and the group (patients versus healthy subjects) for all the thresholds except $0.1 \%$ : at the thresholds $1 \%(\mathrm{p}=0.034 ; \mathrm{F}=3.752), 5 \%(\mathrm{p}=0.013 ; \mathrm{F}$ = 4.927), $10 \%(\mathrm{p}=0.010 ; \mathrm{F}=5.358)$, and 20\% ( $=0.022 ; \mathrm{F}=4.281)$. Repeated-measures ANOVA also demonstrated a significant effect of the tract; the FA values were different between the three tracts (figure 3A). In pairwise analysis, significant tract-by-group interactions were observed between the PT and ILF at the thresholds 5\% $(\mathrm{p}=0.009 ; \mathrm{F}=$ 7.625), $10 \%(\mathrm{p}=0.003 ; \mathrm{F}=10.5)$, and $20 \%(\mathrm{p}=0.007 ; \mathrm{F}=8.3)$ and between the PT and CST at the thresholds $1 \%(\mathrm{p}=0.011 ; \mathrm{F}=7.2), 5 \%(\mathrm{p}=0.007 ; \mathrm{F}=8.4)$, and $10 \%(\mathrm{p}=0.02$; $F=5.9$ ). In other words, compared with the healthy subjects, the degree of impairment was higher in FAPT than in FA ILF and FACST in the patients with MTLE at the thresholds 5\% and $10 \%$ (figure $3 \mathrm{~A}$ ). In contrast, no significant tract-by-group interactions were observed between CST and ILF at any threshold. An example of a significant interaction at the 5\% threshold is shown in figure 3B.

3.2.2 Volume of the tracts and MD were also compared between the patients with MTLE and healthy subjects at each threshold. The average MD of the three tracts was higher in the patients. However, we could not detect any significant tract-by-group 
interactions at any threshold (figure 4A). As expected, the higher the threshold, the smaller the volume of each tract became. No tract-by-group interactions were observed at any threshold (figure 4B).

\subsection{Comparison of white matter integrity using whole brain voxel-wise analysis}

3.3.1 As compared with the healthy control subjects, the whole brain analysis using TBSS revealed a widespread decreased FA in the MTLE patient group (Supplementary figure. 1). The decreased FA was not restricted in the ipsilateral temporal lobe, and distributed in the both hemispheres. The distribution was wider in the hemisphere ipsilateral to the focus than the contralateral hemisphere, and involved the ipsilateral putative propagation tract and the control tracts (ILF, CST).

3.3.2 Increased MD was also observed bilaterally and distributed more widely on the focus side, including the ipsilateral SPT, ILF, and CST in the MTLE patients.

\section{Discussion}

4.1 The present combined study of diffusion tractography and FDG-PET led to two 
main findings. First, we found massive pathways connecting the epileptic focus and remote functional deficit zones in the ipsilateral ventrolateral prefrontal area. These pathways consist mostly of the UF and partly of the AF and the fornix. Second, in MTLE patients, the integrity of these pathways is more prominently impaired than in the two control pathways. The extratemporal glucose hypometabolic region identified by us in the ventrolateral prefrontal cortex ipsilateral to the focus is in good agreement with previous FDG-PET studies in patients with temporal lobe epilepsy (Arnold et al., 1996; Jokeit et al., 1997; Takaya et al., 2006). As we expected in our previous study (Takaya et al., 2009), there is a massive and presumably so exhausted connection through white matter pathways between the epileptic focus and the prefrontal hypometabolic region. Among them, the UF is the dominant pathway, suggesting that UF is a major source of influx that is the cause of the remote functional deficit zone in the prefrontal cortex in patients with MTLE. Two more pathways, the AF and fornix, were also identified. In the present study, the AF could be traced because the seed mask was placed in the temporal hypometabolic region that involves some parts of the middle and superior temporal gyri, which are the cortical terminals of the long segment of the AF (Martino et al., 2013). The other pathway runs through the fornix to the thalamus and then to the prefrontal area. This network is probably related to the hypometabolism and atrophy observed in the ipsilateral thalamus in MTLE 
patients (Bonilha et al., 2010; Khan et al., 1997), although group level analysis did not show significantly decreased glucose uptake in our MTLE group.

4.2 We tested whether a selective or more severe impairment occurs in the PT. For this purpose, we measured FA, a marker of white-matter integrity, along the given tracts using tractography-based analysis. In the MTLE patients, all three tracts (PT, ILF, and CST) showed a significant decrease in FA compared to the control group. This finding is consistent with previous studies on voxel-wise whole brain analysis using TBSS that uncovered a widely distributed FA decrease in the white matter networks ipsilateral to the focus (Focke et al., 2008; Schoene-Bake et al., 2009; Yogarajah et al., 2010). The results were reproduced in our study (Supplementary figure 1). Furthermore, our study extended this finding and demonstrated that, in patients with MTLE, FA in the PT (FAPT) shows a much more significant decrease compared with control tracts (FACST and FAILF), suggesting that white-matter integrity is more severely impaired in the PT.

4.3 We also measured MD, another indicator of microstructural properties of white matter. In contrast to FA, the PT in MTLE patients does not show a specific change in MD compared with the two control tracts. This result may be partly explained by our exclusion of the most severely affected part of the PT in MTLE patients. In fact, another study showed that the most significant increase in MD occurs in the regions adjacent to the focus 
(Focke et al., 2008), which we excluded from analysis in the present study. In addition, water diffusivity in these regions varies depending on the time elapsed since the last seizure (Hufnagel et al., 2003).

4.4 Two possible causes have been proposed as for the change in white-matter integrity in patients with partial epilepsy in relation to epileptic activity: (1) downstream degeneration of output fibers (Concha et al., 2012), and (2) propagation of epileptic activity from the epileptic focus. In our study, the PT is impaired more severely than ILF, even though both tracts originate in the same region (the anterior part of the parahippocampal and fusiform gyri (figure 5). If degeneration downstream of the focus is the main cause of the change in white-matter integrity, the PT and ILF should be damaged to the same extent. Thus, the propagation of epileptic activity is more likely to cause the change in white-matter integrity. Moreover, a decrease in FApT that is not accompanied by a decrease in volume may further support functional but not degenerative nature of the impairment. These lines of evidence suggest that the epileptic activity arising from the focus damages the white matter pathways through which the epileptic activity propagates to a remote functional deficit zone (Takaya et al., 2009). Another possibility for the change in white-matter integrity is that inherent pre-existing abnormalities due to genetic factors or initial precipitating events such as prolonged febrile seizures may also damage the 
specific white matter pathways. And because of the impaired white matter integrity, the frequent propagation of epileptic activity may be facilitated. Longitudinal studies are needed to further examine a direct cause-and-effect relationship between epileptic activity and the change in white matter integrity in patients with MTLE.

4.5 There are some limitations in the present study. First, not all patients underwent surgical focus resection, although all of them had clinically and electrophysiologically diagnosed as MTLE. Second, the sample size is not large enough to analyze patients with left and right MTLE separately. We flipped the images from the right-MTLE patients and used images from all patients for the group analysis to increase statistical power. Accordingly, we also flipped images from the same number of healthy subjects for group comparison between the patients and healthy subjects. This approach allowed us to minimize the influence of physiological differences between hemispheres.

4.6 Despite these limitations, our results shed light on the specific network that is associated with functional changes in remote cortical regions during the resting state in patients with MTLE. Further studies are needed that involve diffusion MRI tractography and task-activation fMRI to assess the role of the PT in the functional impairment during cognitive processing in these remote cortical regions. 


\section{Conclusions}

5.1 Our results showed that the integrity of white matter pathways that connect the epileptic focus with remote functional deficit regions was specifically impaired. Our findings provide insight into the wide range of functional impairment in patients with localization-related epilepsy.

\section{Acknowledgment}

The Ministry of Education, Culture, Sports, Science and Technology of Japan/JSPS

(26282218, 26560465 to Riki Matsumoto and 15H05874 to Akio Ikeda), the Ministry of Health, Labor and Welfare of Japan (H21-023 to Shigetoshi Takaya), the Takeda Science Foundation (to Shigetoshi Takaya), and the Kato Memorial Trust for Nambyo Research (to Riki Matsumoto).

Department of Epilepsy Movement Disorders and Physiology is an endowment department, supported with a grant from GlaxoSmithKline K.K., NIHON KOHDEN CORPORATION, Otsuka Pharmaceutical Co., and UCB Japan Co, Ltd.

\section{Disclosure}

None of the authors has any conflict of interest to disclose. 


\section{REFERENCES}

Arnold, S., Schlaug, G., Niemann, H., Ebner, A., Luders, H., Witte, O.W., Seitz, R.J., 1996. Topography of interictal glucose hypometabolism in unilateral mesiotemporal epilepsy. Neurology 46, 1422-1430.

Behrens, T.E., Berg, H.J., Jbabdi, S., Rushworth, M.F., Woolrich, M.W., 2007. Probabilistic diffusion tractography with multiple fibre orientations: What can we gain? Neuroimage 34, 144-155.

Bonilha, L., Elm, J.J., Edwards, J.C., Morgan, P.S., Hicks, C., Lozar, C., Rumboldt, Z., Roberts, D.R., Rorden, C., Eckert, M.A., 2010. How common is brain atrophy in patients with medial temporal lobe epilepsy? Epilepsia 51, 1774-1779.

Catani, M., Jones, D.K., Donato, R., Ffytche, D.H., 2003. Occipito-temporal connections in the human brain. Brain 126, 2093-2107.

Chassoux, F., Semah, F., Bouilleret, V., Landre, E., Devaux, B., Turak, B., Nataf, F., Roux, F.X., 2004. Metabolic changes and electro-clinical patterns in mesio-temporal lobe epilepsy: a correlative study. Brain 127, 164-174.

Concha, L., Kim, H., Bernasconi, A., Bernhardt, B.C., Bernasconi, N., 2012. Spatial patterns of water diffusion along white matter tracts in temporal lobe 
epilepsy. Neurology 79, 455-462.

Coste, S., Ryvlin, P., Hermier, M., Ostrowsky, K., Adeleine, P., Froment, J.C., Mauguiere, F., 2002. Temporopolar changes in temporal lobe epilepsy - A quantitative MRI-based study. Neurology 59, 855-861.

Dupont, S., Croize, A.C., Semah, F., Hasboun, D., Samson, Y., Clemenceau, S., Baulac, M., 2001. Is amygdalohippocampectomy really selective in medial temporal lobe epilepsy? A study using positron emission tomography with (18)fluorodeoxyglucose. Epilepsia 42, 731-740.

Focke, N.K., Yogarajah, M., Bonelli, S.B., Bartlett, P.A., Symms, M.R., Duncan, J.S., 2008. Voxel-based diffusion tensor imaging in patients with mesial temporal lobe epilepsy and hippocampal sclerosis. Neuroimage 40, 728-737. Hufnagel, A., Weber, J., Marks, S., Ludwig, T., De Greiff, A., Leonhardt, G., Widmann, G., Stolke, D., Forsting, M., 2003. Brain diffusion after single seizures. Epilepsia 44, 54-63.

Jokeit, H., Seitz, R.J., Markowitsch, H.J., Neumann, N., Witte, O.W., Ebner, A., 1997. Prefrontal asymmetric interictal glucose hypometabolism and cognitive impairment in patients with temporal lobe epilepsy. Brain 120 ( Pt 12), 2283-2294. 
Joo, E.Y., Hong, S.B., Han, H.J., Tae, W.S., Kim, J.H., Han, S.J., Seo, D.W., Lee, K.H., Hong, S.C., Lee, M., Kim, S., Kim, B.T., 2005. Postoperative alteration of cerebral glucose metabolism in mesial temporal lobe epilepsy. Brain 128, $1802-1810$.

Khan, N., Leenders, K.L., Hajek, M., Maguire, P., Missimer, J., Wieser, H.G., 1997. Thalamic glucose metabolism in temporal lobe epilepsy measured with 18F-FDG positron emission tomography (PET). Epilepsy Res 28, 233-243.

Love, R., 2005. Two hit hypothesis for temporal lobe epilepsy. Lancet Neurol 4, 458.

Martino, J., De Witt Hamer, P.C., Berger, M.S., Lawton, M.T., Arnold, C.M., de Lucas, E.M., Duffau, H., 2013. Analysis of the subcomponents and cortical terminations of the perisylvian superior longitudinal fasciculus: a fiber dissection and DTI tractography study. Brain Struct Funct 218, 105-121.

Mazziotta, J., Toga, A., Evans, A., Fox, P., Lancaster, J., Zilles, K., Woods, R., Paus, T., Simpson, G., Pike, B., Holmes, C., Collins, L., Thompson, P.,

MacDonald, D., Iacoboni, M., Schormann, T., Amunts, K., Palomero-Gallagher, N., Geyer, S., Parsons, L., Narr, K., Kabani, N., Le Goualher, G., Boomsma, D., Cannon, T., Kawashima, R., Mazoyer, B., 2001. A probabilistic atlas and 
reference system for the human brain: International Consortium for Brain Mapping (ICBM). Philos Trans R Soc Lond B Biol Sci 356, 1293-1322.

Moran, N.F., Lemieux, L., Kitchen, N.D., Fish, D.R., Shorvon, S.D., 2001.

Extrahippocampal temporal lobe atrophy in temporal lobe epilepsy and mesial temporal sclerosis. Brain 124, 167-175.

Oguri, T., Sawamoto, N., Tabu, H., Urayama, S., Matsuhashi, M., Matsukawa, N., Ojika, K., Fukuyama, H., 2013. Overlapping connections within the motor cortico-basal ganglia circuit: fMRI-tractography analysis. Neuroimage 78, 353-362.

Rosenow, F., Luders, H., 2001. Presurgical evaluation of epilepsy. Brain 124, 1683-1700.

Schoene-Bake, J.C., Faber, J., Trautner, P., Kaaden, S., Tittgemeyer, M., Elger, C.E., Weber, B., 2009. Widespread affections of large fiber tracts in postoperative temporal lobe epilepsy. Neuroimage 46, 569-576.

Smith, S.M., Nichols, T.E., 2009. Threshold-free cluster enhancement: addressing problems of smoothing, threshold dependence and localisation in cluster inference. Neuroimage 44, 83-98.

Smith, S.M., Kindlemanny, G., Jbabdi, S., 2013. Cross-Subject Comparison of 
Local Diffusion MRI Parameters. In Diffusion MRI, Second edition

Song, S.K., Sun, S.W., Ju, W.K., Lin, S.J., Cross, A.H., Neufeld, A.H., 2003.

Diffusion tensor imaging detects and differentiates axon and myelin

degeneration in mouse optic nerve after retinal ischemia. Neuroimage 20 ,

1714-1722.

Spanaki, M.V., Kopylev, L., DeCarli, C., Gaillard, W.D., Liow, K., Fazilat, S.,

Reeves, P., Sato, S., Kufta, C., Theodore, W.H., 2000. Postoperative changes in cerebral metabolism in temporal lobe epilepsy. Arch Neurol 57, 1447-1452.

Takaya, S., Hanakawa, T., Hashikawa, K., Ikeda, A., Sawamoto, N., Nagamine, T., Ishizu, K., Fukuyama, H., 2006. Prefrontal hypofunction in patients with intractable mesial temporal lobe epilepsy. Neurology 67, 1674-1676.

Takaya, S., Mikuni, N., Mitsueda, T., Satow, T., Taki, J., Kinoshita, M., Miyamoto, S., Hashimoto, N., Ikeda, A., Fukuyama, H., 2009. Improved cerebral function in mesial temporal lobe epilepsy after subtemporal amygdalohippocampectomy. Brain 132, 185-194.

Velisek, L., Moshe, S.L., 2003. Temporal lobe epileptogenesis and epilepsy in the developing brain: bridging the gap between the laboratory and the clinic. Progression, but in what direction? Epilepsia 44 Suppl 12, 51-59. 
Wakana, S., Caprihan, A., Panzenboeck, M.M., Fallon, J.H., Perry, M., Gollub, R.L., Hua, K., Zhang, J., Jiang, H., Dubey, P., Blitz, A., van Zijl, P., Mori, S., 2007. Reproducibility of quantitative tractography methods applied to cerebral white matter. Neuroimage 36, 630-644.

Yamao, Y., Matsumoto, R., Kunieda, T., Arakawa, Y., Kobayashi, K., Usami, K., Shibata, S., Kikuchi, T., Sawamoto, N., Mikuni, N., Ikeda, A., Fukuyama, H., Miyamoto, S., 2014. Intraoperative dorsal language network mapping by using single-pulse electrical stimulation. Hum Brain Mapp. 35, 4345-4361 Yogarajah, M., Focke, N.K., Bonelli, S.B., Thompson, P., Vollmar, C., McEvoy, A.W., Alexander, D.C., Symms, M.R., Koepp, M.J., Duncan, J.S., 2010. The structural plasticity of white matter networks following anterior temporal lobe resection. Brain 133, 2348-2364. 


\section{FIGURE LEGENDS}

\section{Figure 1}

\section{Group comparison between patients and healthy subjects with FDG-PET and the}

\section{masks in Tractography}

A. A group comparison between patients with mesial temporal lobe epilepsy (MTLE) and healthy subjects. A pronounced hypometabolic region, or a functional deficit zone, was observed both in the temporal and frontal areas ipsilateral to the epileptic focus in the MTLE group. The functional deficit zone was observed not only in the mesial temporal area that involved both the hippocampus and parahippocampus but also in the anterior part of the basal and lateral temporal areas. An extratemporal functional deficit zone was recognized in the ipsilateral ventrolateral prefrontal area. B. The seed and target masks for tracing the putative propagation tract using tractography. Temporal (blue) and extratemporal (green) hypometabolic areas in the Montreal Neurological Institute (MNI) space (left image) were transferred to the individual T1 anatomical space (middle image), and then segmented for the seed and target masks, respectively (right image). Exclusion masks (white) were placed on the midline sagittal plane and the boundary between the two hypometabolic subregions. 


\section{Figure 2}

\section{The Propagation tract at each threshold and the control tracts}

The propagation tract (PT) was traced using probabilistic diffusion tractography. PTs

traced at the thresholds $0.1 \%, 1 \%, 5 \%, 10 \%$, and $20 \%$ of the maximum connectivity value are shown (A). When the threshold was $\geq 5 \%$, the uncinate fasciculus (UF) and the arcuate fasciculus (AF) became predominant, and only the UF remained significant at the $20 \%$ threshold. Control tracts identified at the threshold 5\% are shown in panel $\mathbf{B}$ [corticospinal tract (CST)] and C [inferior longitudinal fasciculus (ILF)]. 


\section{Figure 3}

\section{FA values of CST, ILF, and PT in the MTLE and healthy control groups}

A. Mean and standard deviation (SD) of FACST, FAILF, and FAPT are plotted for five threshold values in the MTLE group (solid line) and the healthy control group (dotted line). Black circles, white squares, and gray triangles denote mean FACST, FAILF, and FAPT, respectively. B. Mean and SD of FACST, FA ILF, and FAPT for the MTLE and control groups at the $5 \%$ threshold. As shown in the tracts traced at this representative threshold, there are significant tract-by-group interactions in the two pairwise analyses: one between ILF and PT $(+; \mathrm{p}=0.009)$ and the other between CST and PT $(++; \mathrm{p}=0.007)$, but there is no interaction between CST and ILF. FAPT decreased more strongly than FAILF and FACST did in the MTLE patients compared with the healthy subjects. The thresholds with significant tract-by-group interactions in the two pairwise analyses are marked with asterisks in figure 5A (5\% and 10\%). The experimental conditions are the same as in figure 2. 


\section{Figure 4}

\section{No significant tract-by-group interactions in mean diffusivity and volume}

A. Mean diffusivity (MD) of the seizure propagation tract (PT), corticospinal tract (CST), and longitudinal fasciculus (ILF) in the mesial temporal lobe epilepsy (MTLE) group and the control group. No significant tract-by-group interactions were observed at any

threshold. B. Volume of PT, ILF, and CST in the MTLE and control group. As the threshold increased, the volume decreased. No significant tract-by-group interactions were observed for the tracts traced at any threshold. The experimental conditions are the same as in figure 3.

\section{Figure 5}

Tracts displayed near the seed mask in representative patients and healthy subjects. A putative seizure propagation tract (PT, red), inferior longitudinal fasciculus (ILF, blue), and corticospinal tract (CST, green) are shown in the axial image at the midbrain level, with the threshold 5\% of the maximum connectivity value. Panel A shows the tracts in six representative patients with left and right MTLE (upper and lower subpanels, respectively). Panel B shows the tracts in six representative healthy controls whose tracts were traced in 
the left and right hemispheres (upper and lower subpanels, respectively). The seed mask encompassed the gray matter of the whole temporal hypometabolic region for both PT and ILF, but the actual seed areas of PT, namely, uncinate fasciculus (UF) and ILF, generally overlapped in the anterior part of the parahippocampal and fusiform gyri.

\section{Supplementary figure}

The whole brain voxelwise statistical analysis of fractional anisotropy (FA) and mean diffusivity (MD) by using Tract Based Spatial Statistics (TBSS). The MTLE patients are compared with the healthy control subjects. The hemisphere ipsilateral to the focus is shown on the left side. In TBSS analysis, the major fasciculi are shown as the mean FA skeleton (green) in the MNI standard space.

A. Parts of the skeleton colored in red correspond to those of significant FA decrease ( $\mathrm{p}<$ 0.01, corrected for family-wise error rate). Decreased FA in the MTLE group is not restricted in the ipsilateral temporal lobe where the epileptic focus is located; Decreased FA is distributed more widely in the hemisphere ipsilateral to the focus than the contralateral hemisphere, and involves both the ipsilateral seizure propagation tract (PT) and the control tracts (ILF, CST). 
Table 1

\begin{tabular}{|c|c|c|c|c|c|c|c|c|c|}
\hline Pt.No. & focus & age & onset age & FS & MRI & Seizure semiology & ictal EEG & spike & ope \\
\hline 1 & $\mathrm{~L}$ & 32 & 22 & - & HS, HA & nausea $\rightarrow$ CPS with dystonic posture of right hand & $\mathrm{L}$ & $\mathrm{L} 80 \%$ & - \\
\hline 2 & $\mathrm{~L}$ & 23 & 19 & - & HS & epigastric rising sensation, ecstacy aura $\rightarrow$ CPS & $\mathrm{L}$ & $\mathrm{L}$ & - \\
\hline 4 & $\mathrm{~L}$ & 26 & 12 & - & HS, HA & nausea, psychic aura $\rightarrow$ CPS & $\mathrm{L}$ & $\mathrm{L}$ & - \\
\hline 5 & $\mathrm{~L}$ & 30 & 22 & + & HS, HA & epigastric rising sensation, deja vu and anxious feeling & $\mathrm{L}$ & $\mathrm{L}$ & + \\
\hline & & & & & & $\rightarrow$ CPS with automatism & & & \\
\hline 7 & $\mathrm{~L}$ & 23 & 22 & + & HS & epigastric rising sensation $\rightarrow$ CPS & $\mathrm{L}$ & - & - \\
\hline 8 & $\mathrm{~L}$ & 35 & 20 & + & HA & abdominal pain $\rightarrow$ CPS with oral automatism & no VEEG & L 95\% & - \\
\hline 9 & $\mathrm{~L}$ & 43 & 15 & + & HA & CPS with automatism, and dystonic posture of right hand & $\mathrm{L}$ & $\mathrm{L}$ & + \\
\hline 10 & $\mathrm{~L}$ & 43 & 19 & - & HA,HS & CPS with automatism, and dystonic posture of right hand & no VEEG & $\mathrm{L}$ & - \\
\hline 11 & $\mathrm{R}$ & 38 & 9 & + & HS, HA & nausea $\rightarrow$ CPS with dystonic posture of left hand & $\mathrm{R}$ & $\mathrm{R}$ & + \\
\hline
\end{tabular}




\begin{tabular}{|c|c|c|c|c|c|c|c|c|c|}
\hline 12 & $\mathrm{R}$ & 45 & 5 & + & HS, HA & epigastric rising sensation, psychic aura $\rightarrow \mathrm{CPS}$ & $\mathrm{R}$ & $\mathrm{R}$ & + \\
\hline 13 & $\mathrm{R}$ & 18 & 12 & + & HS, HA & epigastric rising sensation $\rightarrow$ CPS with automatism and & $\mathrm{R}$ & $\mathrm{R}$ & + \\
\hline & & & & & & dystonic posture of left hand & & & \\
\hline 14 & $\mathrm{R}$ & 23 & 15 & + & HS & CPS with automatism & no VEEG & $\mathrm{R}$ & - \\
\hline 15 & $\mathrm{R}$ & 25 & 24 & + & HS, HA & nausea $\rightarrow$ CPS & $\mathrm{R}$ & $\mathrm{R}$ & - \\
\hline 16 & $\mathrm{R}$ & 24 & 12 & - & HS, HA & CPS with automatism & $\mathrm{R}$ & $\mathrm{R}$ & + \\
\hline 17 & $\mathrm{R}$ & 26 & 4 & - & HS, HA & epigastric rising sensation $\rightarrow$ CPS with automatism & $\mathrm{R}$ & R 90\% & + \\
\hline 18 & $\mathrm{R}$ & 19 & 11 & + & HA & nauseous and uncomfortable feeling $\rightarrow$ CPS & $\mathrm{R}$ & $\mathrm{R}$ & + \\
\hline
\end{tabular}

FC=febrile convulsion, HS=hippocampal sclerosis, HA=hippocampal atrophy, $\mathrm{CPS}=$ complex partial seizure, VEEG=video electroencephalography monitoring 


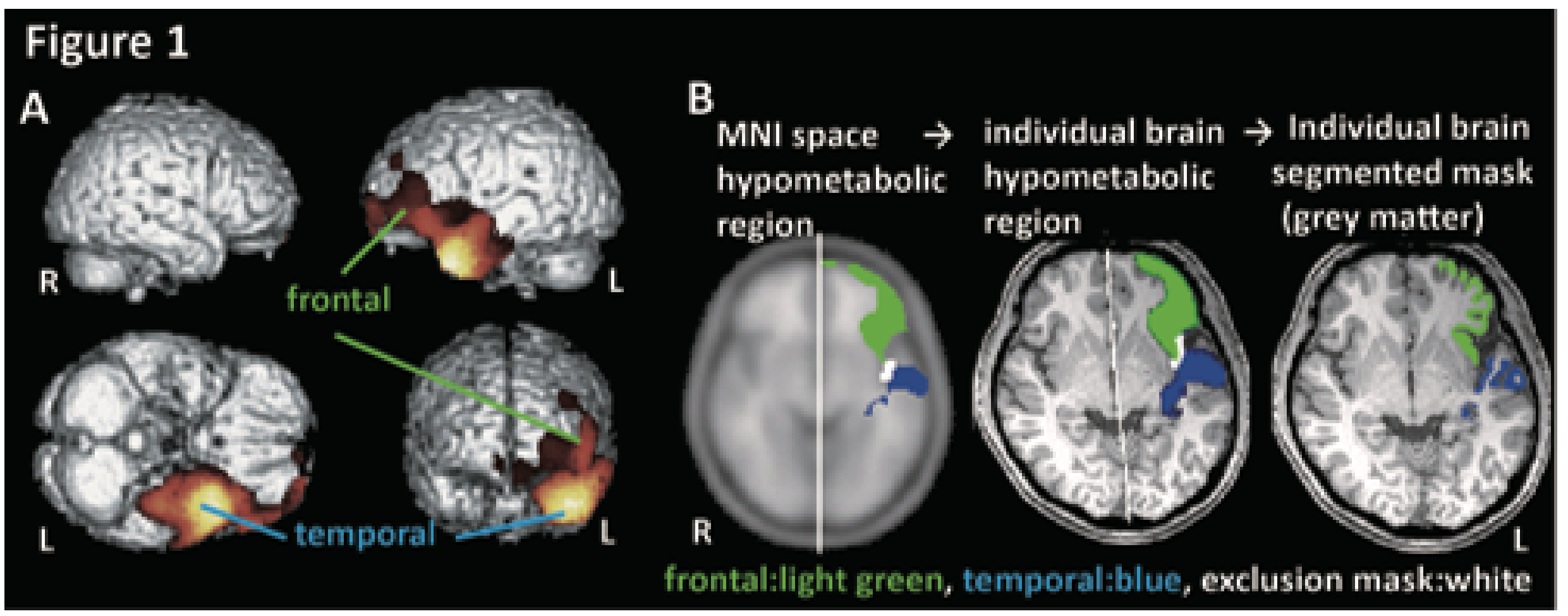




\section{Figure 2 A propagation tract (PT)}

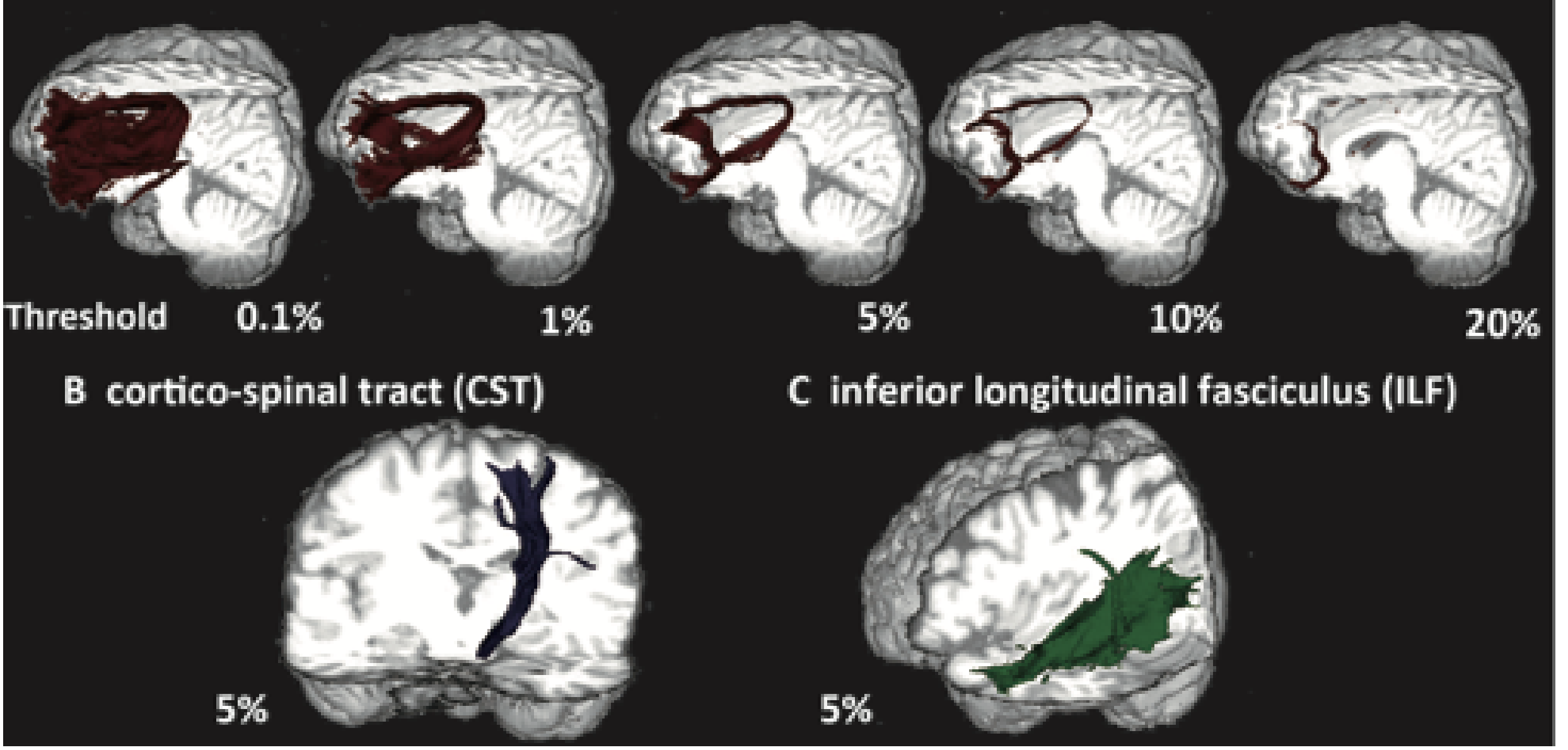


Figure 3

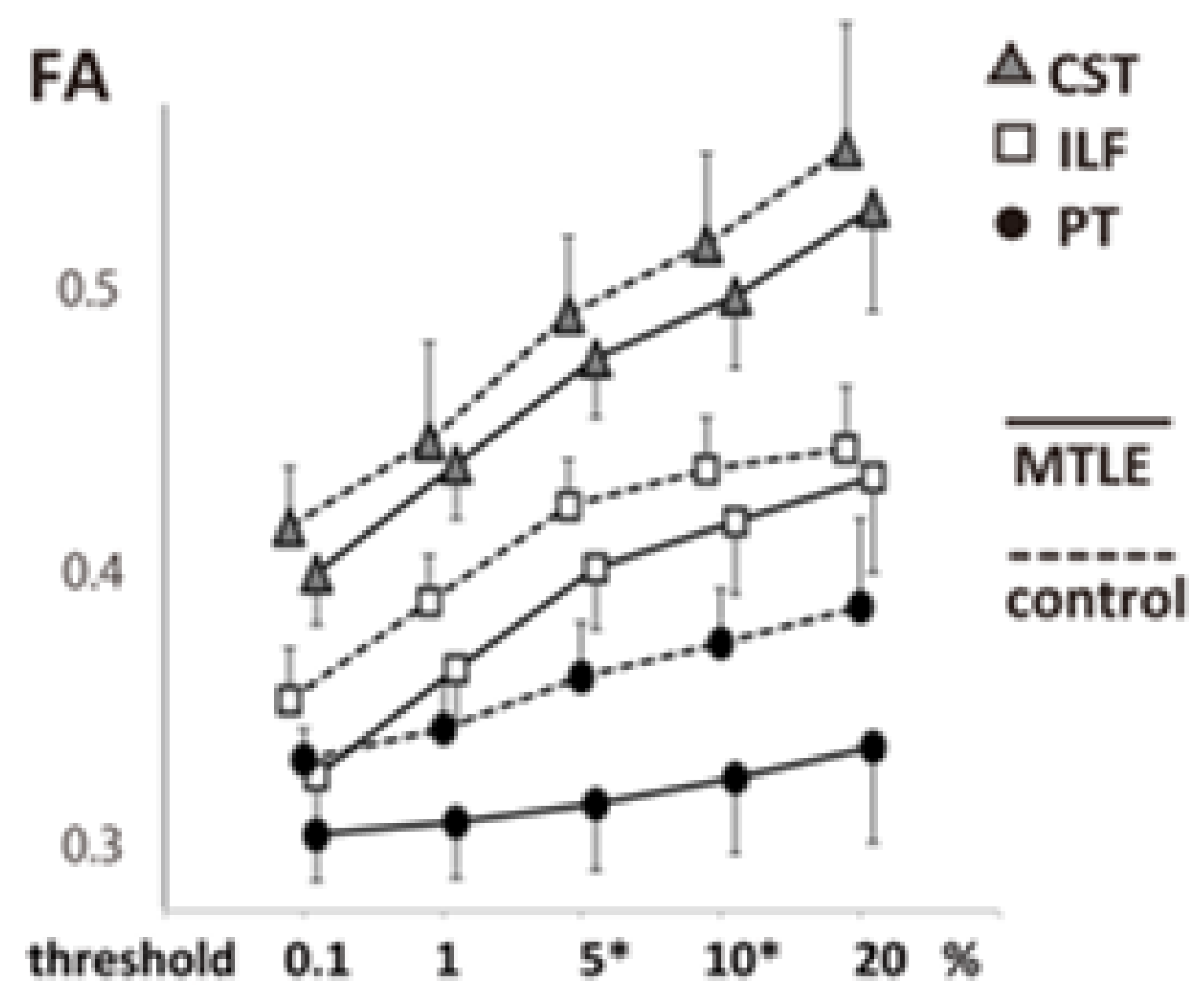

B

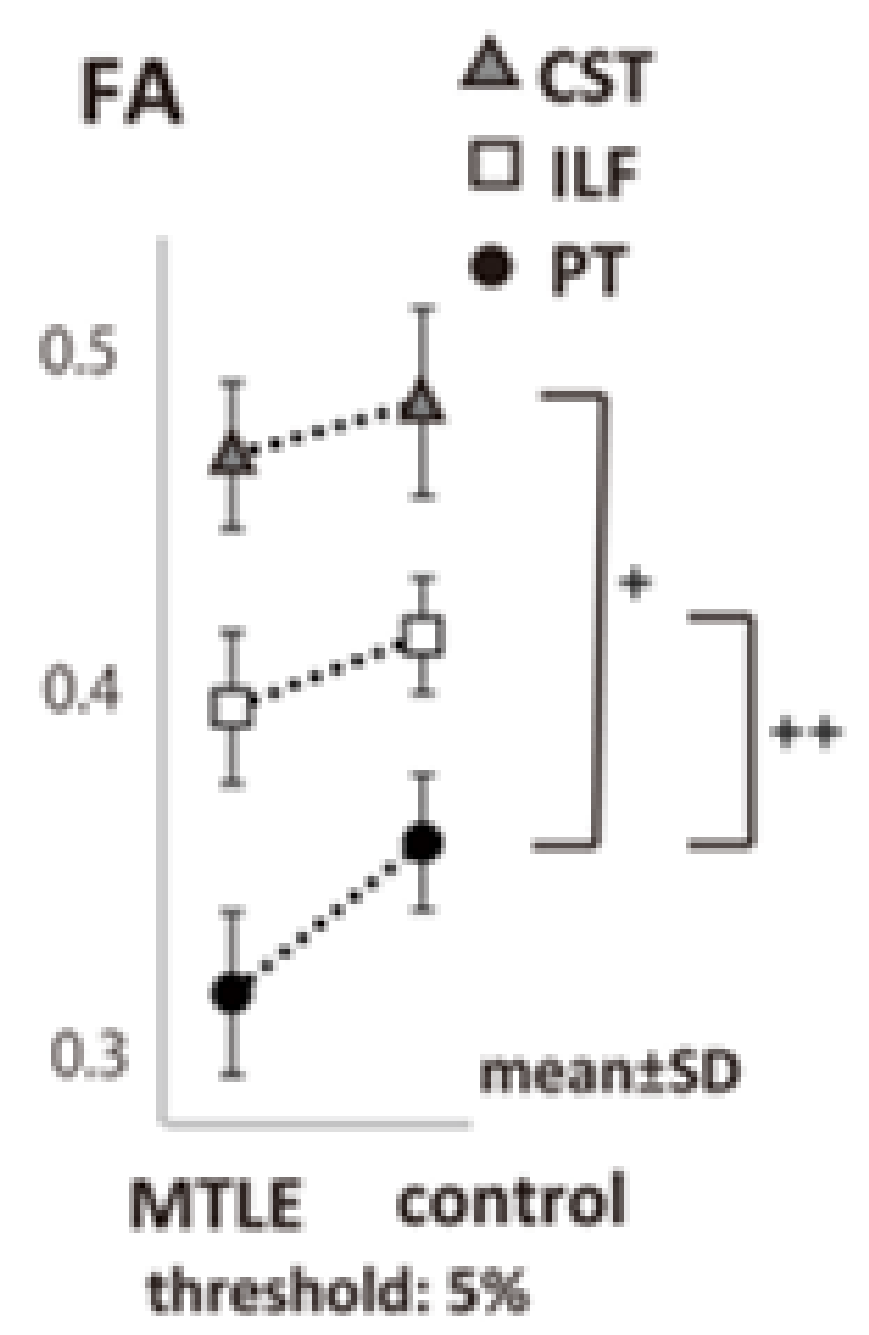


Figure 4

A
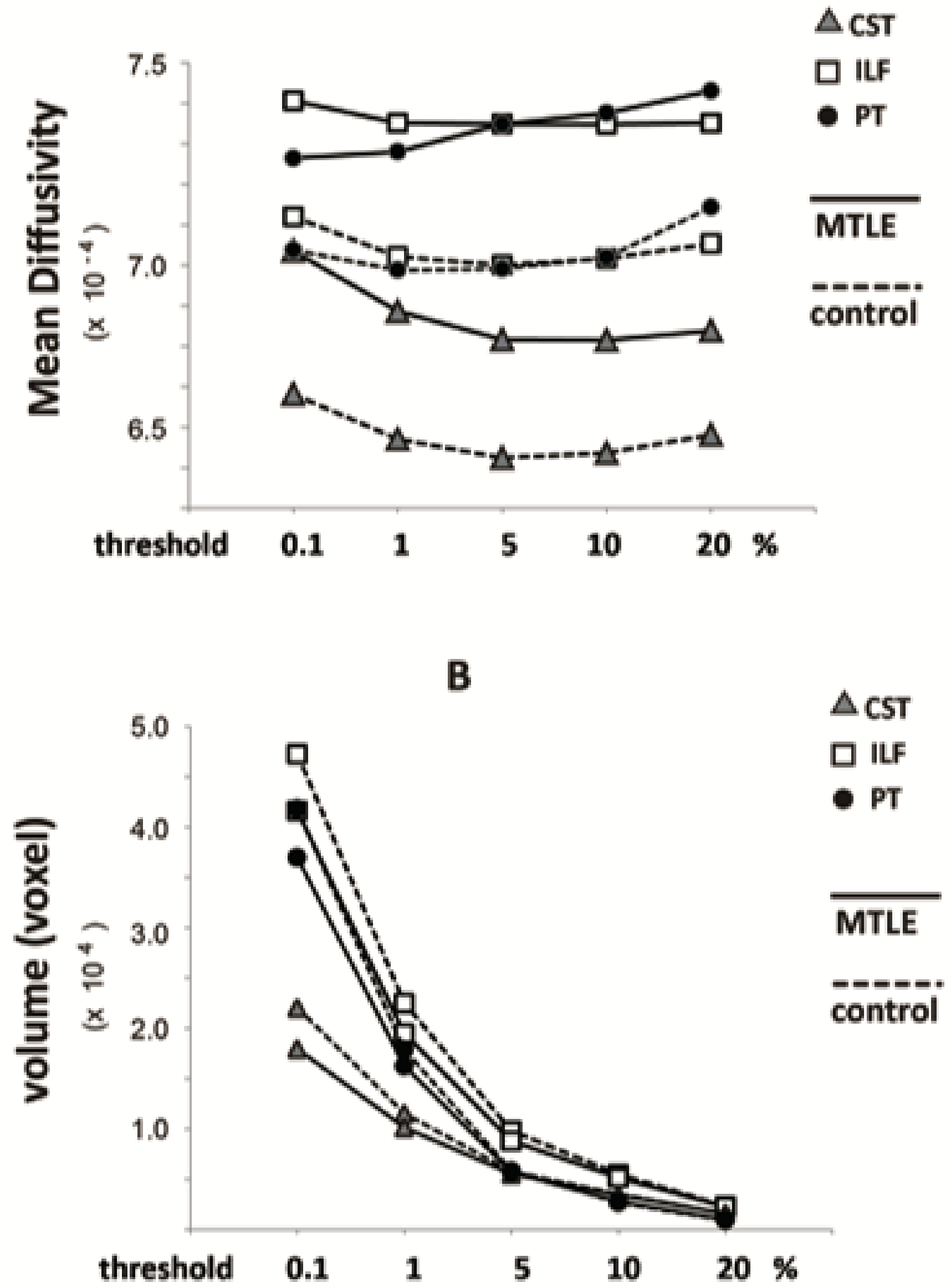
A

Tracts near the seed mask at the midbrain level

Left MTLE

$\mathbf{R}$
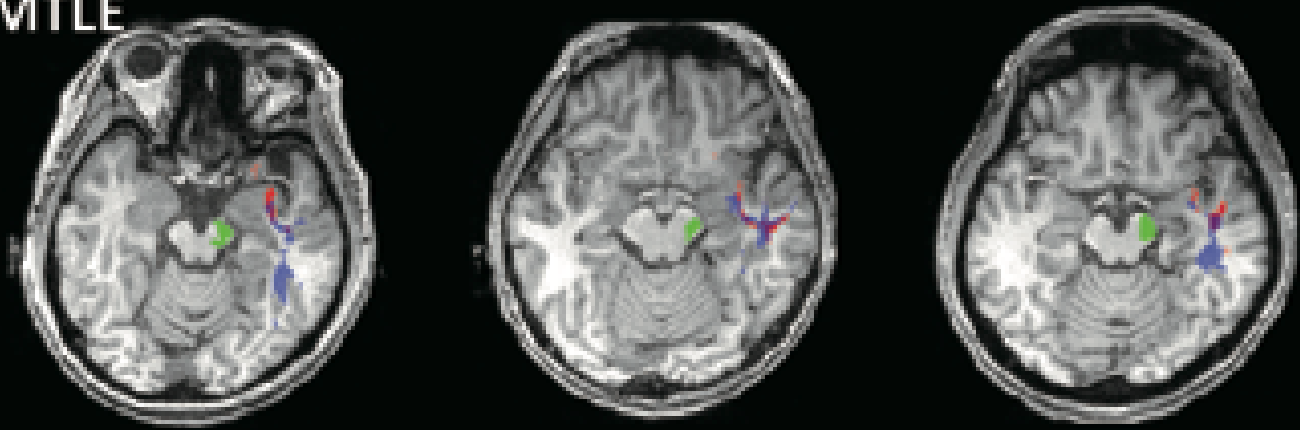

Right MTLE

$\mathbf{R}$
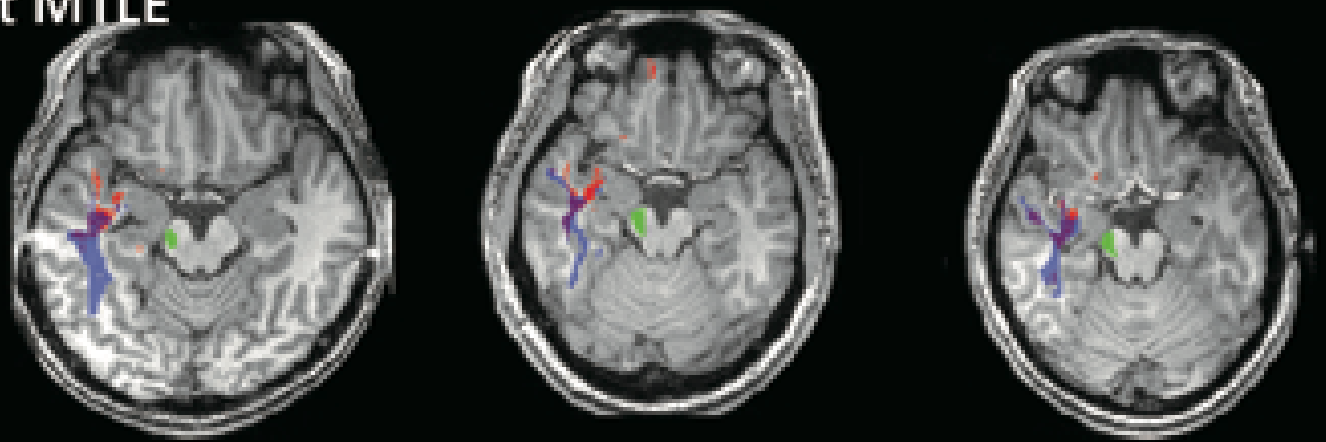

L

B

Healthy controls (analysis in the left hemisphere)

$\mathbf{R}$
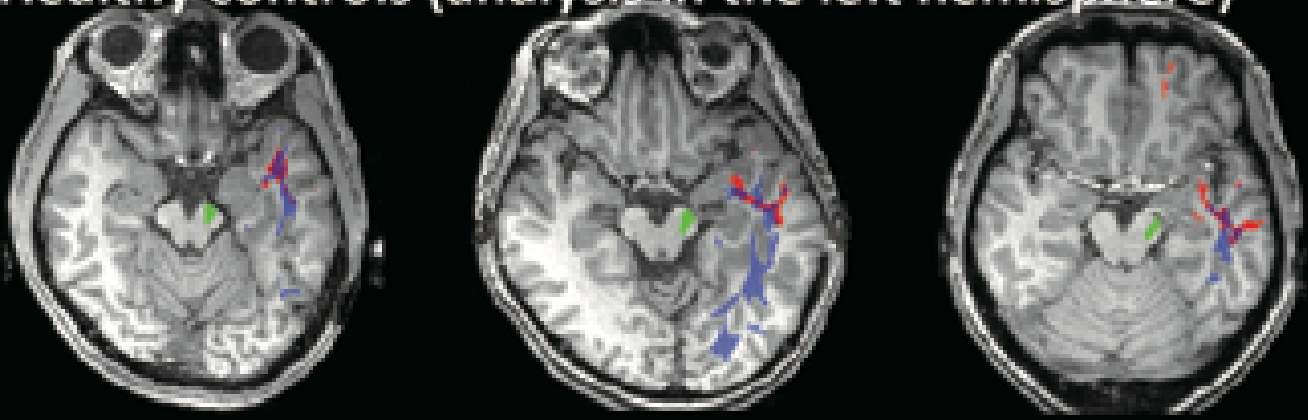

Healthy controls (analysis in the right hemisphere)

$\mathbf{R}$
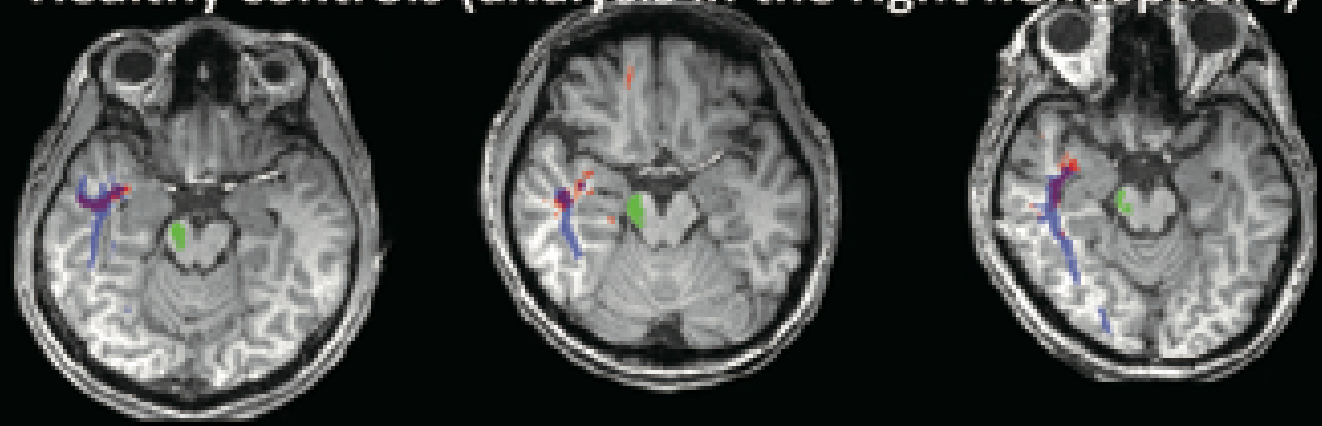

L

PT:red ILFablue CST:green 


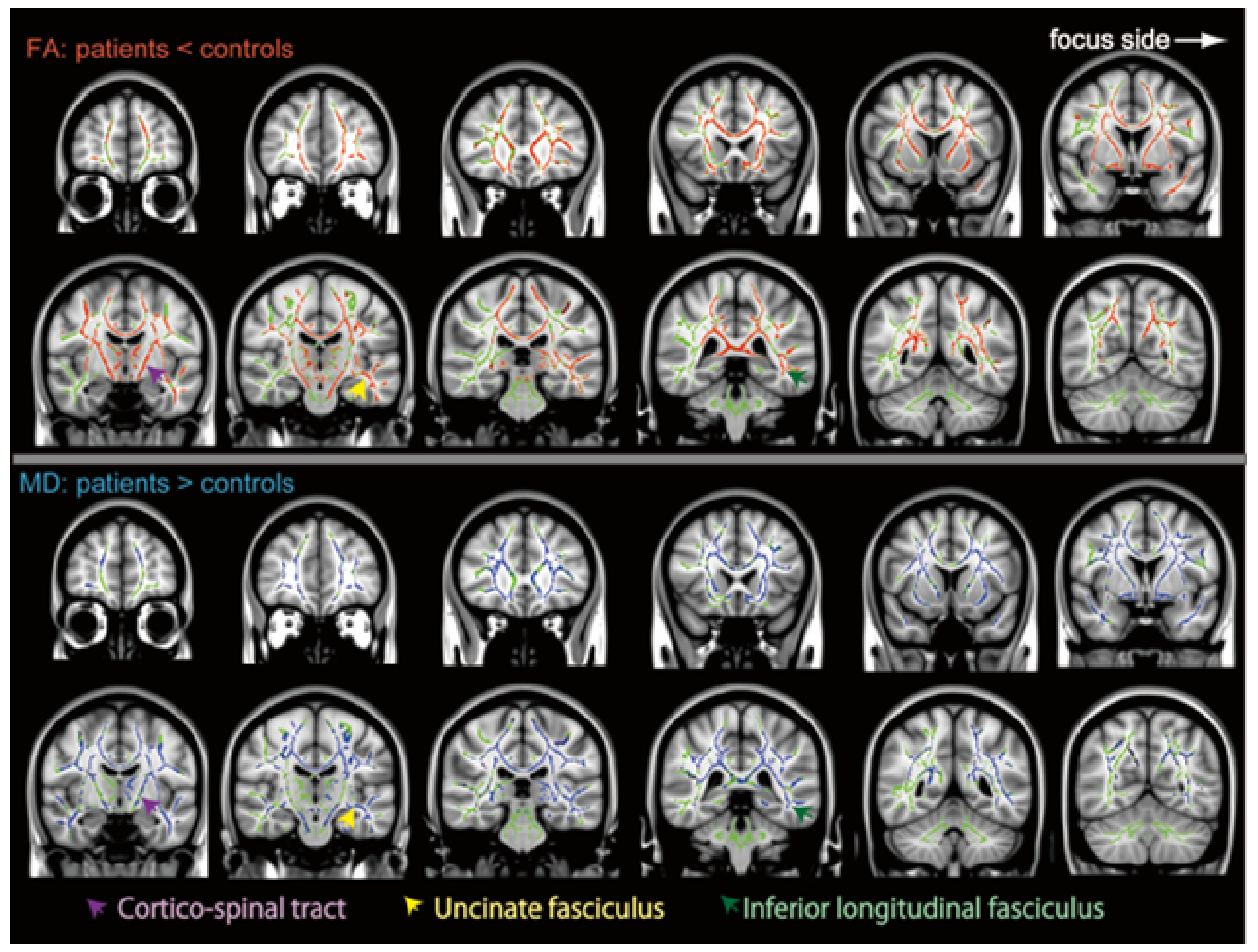

